J. Nonlinear Sci. Appl. 1 (2008), no. 1, $13-19$

The Journal of $\mathbf{N}_{\text {onlinear }} \mathbf{S}_{\text {ciences and its }}$ A pplications

http://www.tjnsa.com

\title{
THE ROLE OF DELAY IN DIGESTION OF PLANKTON BY FISH POPULATION: A FISHERY MODEL
}

\author{
JOYDIP DHAR $^{1 *}$, ANUJ KUMAR SHARMA ${ }^{2}$ AND SANDEEP TEGAR ${ }^{3}$
}

Abstract. In this Paper we have developed a model in which the revenue is generated from fishing and the growth of fish depends upon the plankton which in turn grows logistically. The conditions for the persistence of system around non zero equilibrium have been found out using average Liapnouv function after establishing existence and boundedness of the solution. Then we formulated a model with delay in digestion of plankton by fish. Further the the threshold value of conversional parameter has been found out for hopf-bifurcation. The phenomena of hopf-bifurcation is demonstrated using graphs.

\section{INTRODUCTION}

Many researchers have studied the fishery dynamics with or without considering plankton growth [1, 2, 6, 7, 8, 9, 10, 12]. Again, the delay induced bifurcation in population dynamics shown by many researchers, for example [3, 4, 5, 11, 13, 14]. The first model in the economic theory of open access fishery is as follows [5]:

$$
\begin{aligned}
& \frac{d x}{d t}=r x\left(1-\frac{x}{K}\right)-q E x \\
& T R-T C=p q E x-c E
\end{aligned}
$$

Where $x(t)$ is the population of fish at time $t$ and they are growing logistcally with constant rate ' $r$ ' and ' $K$ ' is the carrying capacity.' $q$ ' is the rate of fishing when effort ' $E$ ' is applied for fishing. Total sustainable revenue $(T R)$ is equal to $p q E x$ Where ' $p$ ' is the per unit cost of harvested biomass. $c E$ is the total $\operatorname{cost}(T C)$ Where $c$ is the cost per unit effort. Sustainable economic rent is the

Date: Received: 27 February 2008; Revised: 18 April 2008.

* Corresponding author.

2000 Mathematics Subject Classification. Primary 92D30, 92D40; Secondary 37G15.

Key words and phrases. Fishery Model, Stability, Delay, Hopf-bifurcation. 
difference of $T R$ and $T C$, i.e., sustainable economic rent is $T R-T C$. 4 From the above model he found out the bio economic equilibrium and concluded that equilibrium level varies around the $M S Y$ (maximum sustainable yield). As a function of cost prise ratio.But the knowledge of bio economic equilibrium is insufficient as it does not gives the answers to the question (1) and what is the optimum effort level; (2) what the optimum sustainable yield and how these can be achieved. However,in order to maximize the sustainable economic $\operatorname{rent}(T R-$ $T C$ ) to achieve the optimum level of fishing effort, he has studied away with the essential ingredient namely the dynamic of economic, which this is a crucial omission. After some time Scheffer [12] gave the autonomous temporal model as:

$$
\begin{gathered}
\frac{d x}{d t}=r x\left(1-\frac{x}{K}\right)-q E x \\
\frac{d E}{d t}=k E(p q x-c)
\end{gathered}
$$

Though the above model contains the dynamics of economy and biological process. But it does not contain the dynamic of plankton species which provide the necessary nutrients for the growth of fish, as well as the above model is not having the equation of economic rent. The above mentioned omission in the model have forced us to formulate a model which include the dynamics of plankton which grow logistically. Moreover we shall be using the dynamic of economic rent in term of effort.

\section{The Mathematical Model}

Model is assumed to be closed in which plankton species are growing logistically with a growth rate $a$ and has the carrying capacity $k$. Again, $\alpha$ is the rate of harvesting of plankton spices by the fish population and the interaction between the plankton and fish is assumed to follow law of mass action. Conversion rate from plankton to fish is denoted by $\alpha_{1}$, takeing $\beta=\alpha \alpha_{1}$ is the conversion rate of the fish population. The self decay of fish population is denoted by $c_{1}$. The rate of catchabliety of fish when effort $E$ is applied is denoted by $q_{1}$. The cost per unit fish $b_{1}$ and $c$ is the cost per unit effort All the parameters are assumed to be positive. The rate of change of economic rent $\left(E_{R}\right)$ is equal to the difference of total revenue from fish sale and total cost of fishing. now $P(t), F(T)$ and $E(T)$ represent plankton population, fish population and effort respectively at any time $t$. Hence, we can write the mathematical model for the above system as follows:

$$
\begin{gathered}
\frac{d P}{d t}=a P\left(1-\frac{P}{K}\right)-\alpha P F \\
\frac{d F}{d t}=\beta P F-c_{1} F-q_{1} E F \\
\frac{d E_{R}}{d t}=q_{1} b_{1} E F-c E
\end{gathered}
$$


Taking $E_{R}=\rho E$ and non-dimensionalized the above system and choosing the following new variables:

$$
x \equiv \frac{P}{K}, y \equiv \frac{F}{F_{0}}, z \equiv \frac{E}{E_{0}} a t \equiv \tau .
$$

The above system reduces to

$$
\begin{gathered}
\frac{d x}{d t}=x(1-x)-b x y \\
\frac{d y}{d t}=\beta_{1} x y-c_{0} y-y z \\
\frac{d z}{d t}=\theta y z-d z
\end{gathered}
$$

Where

$$
b=\frac{\alpha F_{0}}{a} ; \beta_{1}=\frac{\beta k}{a} ; c_{0}=\frac{c_{1}}{a} ; \theta=\frac{b F_{0}}{\rho E_{0}} ; d=\frac{c}{\rho a} .
$$

\section{Existence of Equilibrium Points and Boundedness}

There are four feasible equilibria of the system (2.4)-(2.6), namely,

(1) $E_{0}=(0,0,0)$ is the trivial steady state,

(2) $E_{1}=(1,0,0)$, here only plankton population exists,

(3) $E_{2}=\left(\frac{c_{0}}{\beta_{1}}, \frac{1}{b}\left(1-\frac{c_{0}}{\beta_{1}}\right), 0\right)$, here no fishing take place only plankton and fish are living together and

(4) (iv) $E^{*}=\left(x^{*}, y^{*}, z^{*}\right)$, all three population co-exists, where $x^{*}=1-\frac{b d}{\theta}$; $y^{*}=\frac{d}{\theta} ; z^{*}=\beta_{1}-c_{0}-\frac{\beta_{1} b d}{\theta}$.

Again, $E_{2}$ is feasible if $\beta_{1}>c_{0}$ and $E^{*}$ is feasible if

$$
\theta>\max \left\{b d, \frac{\beta_{1} b d}{\beta_{1}-c_{0}}\right\} \text { and } \beta_{1}>c_{0} .
$$

Now we will show that all the solutions of the system (2.4)-(2.6) are bounded in a region $B \subset R_{+}^{3}$. We consider the following function

$$
w(\tau)=x(\tau)+y(\tau)+z(\tau)
$$

Then the time derivative of the above function after substituting the values from (2.4)- 2.6 , we get

$$
\begin{gathered}
\frac{d w}{d \tau}=x(1-x)-\left(d-\beta_{1}\right) x y-(1-\theta) y z-d z-c_{0} y \\
\frac{d w}{d \tau} \leq x(1-x)-d z-c_{0} y . \\
\frac{d w}{d \tau}+\eta w(\tau) \leq(1+\eta) x-x^{2}=f(x) .
\end{gathered}
$$

where

$$
\eta=\min \left\{d, c_{0}\right\} \text { and } f(x)=(1+\eta) x-x^{2} .
$$


Hence

$$
\frac{d w}{d \tau}+\eta w(\tau) \leq \frac{1+\eta^{2}}{4}=M(\text { say })
$$

Now, using comparison theorem, as $\tau \rightarrow \infty$, then

$$
\sup w(\tau) \leq \frac{M}{\eta}
$$

\section{Dynamical Behavior}

The dynamical behavior of the equilibrium can be studied by computing the variational matrix at various equilibrium points and Using the Routh-Hurwitz criterion. We can note the following points:

(1) The equilibrium point $E_{0}$ is a saddle point with locally stable manifold in $\mathrm{Y}-\mathrm{Z}$ plane and with unstable manifold in $\mathrm{x}$ direction.

(2) If $\beta_{1}<c_{0}$, then the equilibrium point $E_{1}(1,0,0)$ is locally asymptotically stable in X-Y-Z space, as $E_{2}$ and $E_{3}$ does not exit for $\beta_{1}<c_{0}$, but if $\beta_{1}>c_{0}$, then $E_{1}$ is saddle point with local stable manifold in $\mathrm{X}-\mathrm{Z}$ direction and with unstable manifold in Y-direction.

(3) $E_{2}$ is saddle point but with stable manifold in X-Y plane when $\beta_{1}>c_{0}$.

Lemma 4.1. If $\beta_{1}>c_{0}$, then $E_{2}$ is globally asymptotically stable in the interior of positive quadrant of $X$ - $Y$ plane.

Proof. Taking $H=\frac{1}{x y}$, where is $H>0$ in the interior of positive quadrant and

$$
\begin{gathered}
f_{1}(x y)=x(1-x)-b x y \\
f_{2}(x y)=\beta_{1} x y-c_{0} y .
\end{gathered}
$$

Clearly

$$
\frac{d f_{1} H}{d x}+\frac{d f_{2} H}{d y}<0
$$

and it does not changes sign in positive quadrant. Therefore, using BendixonDulec criterion there does not exist any limit cycle in $\mathrm{X}-\mathrm{Y}$ plane.

Now we will study the uniform persistence of the system using average Lyapunove function [15].

Theorem 4.2. The system (2.4)-(2.6) is uniformly persistent if $l_{1}>c_{0} l_{2}+d l_{3}$, $l_{2}>\frac{d l_{3}}{\beta_{1}-c_{0}}$ and $\beta_{1}>c_{0}$, where $l_{1}, l_{2}, l_{3}$ are all positive.

Proof. Take average Lyapunove function for the system as $\rho(X)=x_{1}^{l} y_{2}^{l} z_{3}^{l}$. Clearly $\rho(x)$ is non negative function defined in $R_{+}^{3}$ and $\mathrm{X}$ is a function of $x, y, z$. After differentiating we have

$$
\begin{aligned}
\psi(X) & =\frac{\dot{\rho}(X)}{\rho(X)}=l_{1} \frac{\dot{x}}{x}+l_{2} \frac{\dot{y}}{y}+l_{3} \frac{\dot{z}}{z} \\
& =l_{1}(1-x-b y)+l_{2}\left(\beta_{1} x-c_{0}-z\right)+l_{3}(\theta y-d)
\end{aligned}
$$

Further from above theorem, the system has no periodic orbit in the interior of $\mathrm{X}-\mathrm{Y}$ plane. Thus, the uniform persistent exists, if there exists $l_{1}, l_{2}$ and $l_{3}$, such that $\psi(X)$ is positive at $E_{0}, E_{1}$ and $E_{2}$. Now 
(1) $\psi\left(E_{0}\right)>0$ if $l_{1}>c_{0} l_{2}+d l_{3}$

(2) $\psi\left(E_{1}\right)>0$ if $l_{2}>\frac{d l_{3}}{\beta_{1}-c_{0}}$

(3) $\psi\left(E_{2}\right)>0$ if $\theta\left(1-\frac{c_{0}}{\beta_{1}}\right)>d \Rightarrow \beta_{1}>c_{0}\left(1-\frac{d}{\theta}\right)$, which is always true for any set of positive values of $l_{1}, l_{2}, l_{3}$, since in the existence of $E_{2}, \beta_{1}>c_{0}$ and all the parameter of the systems are positive.

(4) $\psi\left(E_{3}\right)>0$, always true for any set of positive values of $l_{1}, l_{2}, l_{3}$.

Hence, the system is persistent if

$$
l_{1}>c_{0} l_{2}+d l_{3}, l_{2}>\frac{d l_{3}}{\beta_{1}-c_{0}} \text { and } \beta_{1}>c_{0}
$$

are satisfied.

Example 4.3. Let us choose suitable values of the parameters: $b=0.5, c_{0}=0.01$, $\theta=0.5, d=0.1, l_{1}=0.5, l_{2}=0.3$ and $l_{3}=0.5$, which will clearly satisfy the conditions (4.1). Hence the system always stable around $E^{*}$. The numerical solution of the system (2.4)-(2.6), taking the same set of values for the parameters as mentioned above, with $\beta_{1}=0.02,0.03$ and 0.2 respectively.

\section{The Model with Delay}

Here we assume that fishes takes time to digest the plankton and grow proportionally.

$$
\begin{gathered}
\frac{d x}{d t}=x(1-x)-b x y \\
\frac{d y}{d t}=\beta_{1} y \int_{-\infty}^{t} \beta \exp \left(-\beta(t-s) f(s)-c_{0} y-y z\right.
\end{gathered}
$$

Where $f(s)=f(x)=x$

Put

$$
\frac{d z}{d t}=\theta y z-d z
$$

$$
R(t)=\int_{-\infty}^{t} \beta \exp (-\beta(t-s) f(s) d s
$$

Therefore

$$
\begin{gathered}
d R / d t=\beta(x-R) \\
\frac{d x}{d t}=x(1-x)-b x y \\
\frac{d y}{d t}=\beta_{1} y R-c_{0} y-y z \\
\frac{d z}{d t}=\theta y z-d z
\end{gathered}
$$

There are three steady state of the system with delay, namely,

(1) $E_{0}(0,0,0,0)$ is trivial equilibrium,

(2) $E_{1}(1,0,0,0)$ here only plankton population exists 
(3) $E^{*}\left(x^{*}, y^{*}, z^{*}, R^{*}\right)$ is the non-trivial equilibrium, where $x^{*}=(1-b d / \theta)$, $y^{*}=b / d, z^{*}=\left(\beta_{1}-c_{0}-b \beta_{1} d / \theta\right)$ and $R^{*}=(1-b d / \theta)$.

The non-trivial equilibrium is non negative if $(1-b d / \theta) \beta_{1}>c_{0}$ and $\left.\theta\right)>b d$ the characteristic equation of the delayed system at $E-{ }^{*}\left(x^{*}, y^{*}, z^{*}, R^{*}\right)$ is given by

$$
\lambda^{4}+A_{1} \lambda^{3}+A_{2} \lambda^{2}+A_{3} \lambda+A_{4}=0
$$

Where $A_{1}=\beta+x^{*}, \quad A_{2}=\theta y^{*} z^{*}+\beta x^{*}, \quad A_{3}=\left(\beta+x^{*}\right) \theta y^{*} z^{*}+\beta \beta_{1} b x^{*} y^{*}$ and $A_{4}=\theta \beta x^{*} y^{*} z^{*}$ on substitution the values of $x^{*}, y^{*}, z^{*}$ and $R^{*}$, it can be easily verified that $A_{i}>0$, for $i=1,2,3,4$. Now, from Routh-Hurwitz criterion a set of necessary conditions for all the roots of the equation (5.7) having negative real part are $A_{i}>0, i=1,2,3,4$. Now we shell diagnose the hopf bifurcation of the given system for $\beta_{1}$ variable which represent the conversional rate from plankton to fish population.

We know that the necessary and sufficient conditions for Hopf-Bifurcation, that there exist $\beta_{1}=\beta_{0}$ such that (i) $A_{i}\left(\beta_{0}\right)>0$ for $i=1,2,3$, 4 , (ii) $H_{2}\left(\beta_{0}\right)=$ $A_{1} A_{2}-A_{3} \neq 0$, (iii) $H_{3}\left(\beta_{0}\right)=A_{1} A_{2} A_{3}-A_{1}^{2} A_{2}-A_{3}^{2}=0$ and (iv) $\frac{d H_{3}}{d \beta_{1}}\left(\beta_{0}\right) \neq 0$.

The condition (i) is true for all values of $\beta_{1}$ established earlier. Now, assume there exist $\beta_{0}>0$ such that $H_{3}\left(\beta_{0}\right)=0$, which implies

$$
a_{1}+a_{2} \beta_{0}+a_{3} \beta_{0}^{2}=0
$$

where $a_{1}=\left(\beta+x^{*}\right) L_{1} L_{3}-\left(\beta+x^{*}\right)^{2} L_{5}-L-3^{2}, a_{2}=\left(\beta+x^{*}\right)\left(L_{1} L_{4}-L_{2} L_{3}\right)-$ $L_{6}\left(\beta+x^{*}\right)^{2}-2 L_{3} L_{4}, a_{3}=\left(\beta+x^{*}\right) L_{2} L_{4}-L_{4}^{2}, L_{1}=\beta x^{*}-\theta y^{*} c_{0}, L_{2}=\theta x^{*} y^{*} L_{3}=$ $-\left(\beta+x^{*}\right) \theta y^{*} c_{0}, L_{4}=\beta b x^{*} y^{*}+\left(\beta+x^{*}\right) \theta x^{*} y^{*} L_{5}=-\theta c_{0} \beta b x^{*} y^{*}, L_{6}=\theta \beta b\left(x^{*}\right)^{2} y^{*}$. By taking $c_{0}=0.01, b=0.5, \theta=0.5, d=0.1$ from $(5.8)$, we get $\beta_{0}=0.0216$. Further, $\left.H_{2}\left(\beta_{0}\right)=\beta x^{*}(\beta+1-b d / \theta)-\beta_{1} b^{2} d\right) \neq 0$ and $\frac{d H_{3}}{d \beta_{1}}\left(\beta_{0}\right) \neq 0$. Hence the system start bifurcating at $\beta_{0}$. The results are shown graphically, taking $b=0.5$, $c_{0}=0.01, \theta=0.5, d=0.1, \beta=0.01$ with same set of values of $\beta_{1}$ as in the previous section. When $\beta_{1}=0.02<\beta_{0}=0.0216$, the solution is converging to $E^{*}$ and as $\beta_{1}$ crosses the value $\beta_{0}$, the system converges in a limit cycle, which is shown in Figure 2(b) and 2(c). On comparing the figures 1(a)-(c) with 2(a)-(c) respectively, it can be established that the delay induces Hopf-bifurcation in the system.

Remark 5.1. In this model we have established using average Liapounove function that the model without delay in conversion rate of fish persist uniformly under some conditions. Again, with the introduction of delay in conversion rate of fish, the system starts oscillating when $\beta_{1}$ crosses a threshold value as shown in figures.

\section{REFERENCES}

1. L.G.Anderson, Optium economic yield of a fishery given a variable prise of output, J. Fishries Reasearch Board of Canada, 30, (1973), 509-518. 1

2. J. Blaxter and A. Southward, Advances in marine biology, Academic Press, London, 1997.

11 
3. J. Chattopadhyay, R. Sarkar and A. E. Abdllaoui, A delay differential equation model on harmful algal blooms in the presence of toxic substances, IMA J. Math. Appl. Med. Biol., 19, (2002), 137-161. 1

4. J Chattopadhyay, R. Sarkar and S. Mandal, Toxinproducing plankton may act as a biological control for planktonic blooms field study and mathematical modelling, J.Theor. Biol., 215, (2002), 333-344. 1

5. C.W. Clark, A delayed recruement model of population dynamics, with an application tob bleen the whale poplation, J. Mathematical Biology, 31, (1976), 381-391. 1

6. C.W. Clark, Mathematical Bioeconomics: The Optimal Management of Renewable resources, John Wiley and Sons, New York, 1990. 1

7. E. Graneli et. al., From anoxia to fish poisoning: The last ten years of phytoplankton blooms in swedish marine waters: in Novel phytoplankton blooms, (eds) E Cosper et. al. (Springer) pp. 407-427, 1989. 1]

8. D. Ludwing and J. Varha, Optimal harvesting of randomly fluctuing resource. Numerical methods and results, SIAM J. Applied Mathematics, 37, (1979), 185-205. 1

9. H. Malchow, B. Radtke, M. Kallache, A. Medvinsky and S. Tikhonov Dand Petrovskii, Spatio-temporal pattern formation in coupled models of plankton dynamics and fish school motion, J. Nonlinear Analysis: Real World Applications, 1, 2000, 53-67. 1

10. Monovoisin, C. GandBolito and J.G. Ferreira, Fish Dynamics in a coastal food chain, Bol. inst Esp Oceanoger, (1999),431-440. 1

11. R. Sarakar and H. Malchow, Nutrients and toxin producing phytoplankton control algal blooms a spatio-temporal study in a noisy environment, J. Biosci. 30(5), (2005), 749-760. 1 .

12. M. Scheffer, Fish and nutrients interplay determines algal biomass, Oikos 62, (1991), 271282. 1. 1.

13. J. Truscott and J. Brindley, Ocean plankton populations as excitable media, Bull. Math. Biol., 56, (1994), 981-998. 1

14. S. Wiggins, Interoduction to Applied non linear Dyanamical System and Chaos, Springer Verlag, New york, 1990. 1

15. Li Zhan, Shun Zhisheng and Ke Wang, Persistence and extinction of single population in a polluted environment, Electronic J. Differential Equations, 108, (2004), 1-5. 4

${ }^{1}$ Department of Applied Sciences, ABV-Indian Institute of Information TechNOLOGY AND Management, Gwalior-474 010, M.P, INDIA.

E-mail address: jdhar@iiitm.ac.in

2 Department of Mathematics, L.R.D.A.V. College, Jagraon-142026, Ludhiana, PUNJAB, INDIA.

E-mail address: anujsumati@gmail.com

3 School of Mathematics and Allied Sciences, Jiwaji University, Gwalior474011, M.P., INDIA.

E-mail address: tegarss@yahoo.com 